\title{
Brief Report: Reduced Grouping Interference in Children with ASD: Evidence from a Multiple Object Tracking Task
}

\author{
Kris Evers • Lee de-Wit • Ruth Van der Hallen • \\ Birgitt Haesen • Jean Steyaert • Ilse Noens • \\ Johan Wagemans
}

(C) Springer Science+Business Media New York 2014

\begin{abstract}
This study was inspired by the more locally oriented processing style in autism spectrum disorders (ASD). A modified multiple object tracking (MOT) task was administered to a group of children with and without ASD. Participants not only had to distinguish moving targets from distracters, but they also had to track targets when they were visually grouped to distracters, a manipulation which has a detrimental effect on tracking performance in adults. MOT performance in the ASD group was also affected by grouping, but this effect was significantly reduced. This result highlights how the reduced bias towards more global processing in ASD could influence
\end{abstract}

K. Evers $(\bowtie) \cdot$ L. de-Wit · R. Van der Hallen · B. Haesen J. Wagemans

Laboratory of Experimental Psychology, KU Leuven,

Tiensestraat 102, Box 3711, 3000 Leuven, Belgium

e-mail: kris.evers@psy.kuleuven.be

\section{K. Evers · J. Steyaert}

Department of Child Psychiatry, UPC-KU Leuven, Leuven, Belgium

K. Evers · R. Van der Hallen · B. Haesen ·

J. Steyaert · I. Noens · J. Wagemans

Leuven Autism Research (LAuRes), KU Leuven,

Leuven, Belgium

J. Steyaert

Department of Clinical Genetics, University Hospital Maastricht,

Maastricht, The Netherlands

I. Noens

Parenting and Special Education Research Unit, KU Leuven, Leuven, Belgium

I. Noens

Psychiatric and Neurodevelopmental Genetics Unit,

Massachusetts General Hospital, Boston, MA, USA further stages of cognition by altering the way in which attention selects information for further processing.

Keywords Attention - Autism spectrum disorder (ASD) . Global interference $\cdot$ Grouping $\cdot$ Multiple object tracking · Weak central coherence

\section{Introduction}

Atypical perceptual organization in individuals with an autism spectrum disorder (ASD) seems to be characterized by superior local processing and a reduction in the automatic integration of local information into global wholes. In a seminal study by Shah and Frith (1983), matched comparison samples were outperformed by children with ASD when asked to detect a target shape embedded within a larger figure (for a recent review of the use of the embedded figures test in ASD, see White and Saldana 2011). Later studies with ASD samples supported this finding using, for instance, visual illusions (Happé 1996), hierarchical figures (Plaisted et al. 1999), the Rey-Osterrieth complex figure task (Tsatsanis et al. 2011), and block design tasks (Shah and Frith 1993). However, many researchers have failed to replicate a local processing bias or a deficit in gist perception in participants with ASD, resulting in a highly inconsistent literature (for a review, see Behrmann et al. 2006; Dakin and Frith 2005; Simmons et al. 2009). In line with a large body of mixed empirical work, recent versions of the weak central coherence theory (WCC; Frith and Happé 1994; Happé and Booth 2008; Happé and Frith 2006) and the Enhanced Perceptual Functioning hypothesis (EPF; Mottron and Burack 2001; Mottron et al. 2006) have abandoned the global deficit account. Instead, both neurocognitive theories have shifted 
towards a description in terms of an atypical perceptual bias, with a disinclination for global (e.g., Koldewyn et al. 2013) or a preference for local processing. Moreover, some authors have argued that the atypical processing style in individuals with ASD is not merely a bottom-up perceptual phenomenon (Mottron et al. 2013; Samson et al. 2012), but that high-level cognitive processes, such as attention, also play an important role in the bias towards a more local processing level (Loth et al. 2010; Mitchell et al. 2010).

The current work was inspired by this more local default setting in ASD, and by the important role of objects in perception and cognition. By modifying a standard multiple object tracking (MOT) task (Pylyshyn and Storm 1988), Scholl et al. (2001) demonstrated the compelling and automatic influence of the perceptual organization of a display (into objects) on the allocation of attention (for a discussion about the relationship between perception and attention, see Driver et al. 2001). This finding was brought to light by adapting the standard MOT task such that participants not only had to simultaneously distinguish moving targets from distracters, but they also had to track these targets when they were visually grouped to distracters (Scholl et al. 2001). Connecting targets with distracters, thereby visually grouping them into an object, caused participants to lose track of the individual targets. These studies offered a convincing demonstration of the obligatory (non-strategic) influence of target merging or grouping, even though grouping was detrimental to task performance. Scholl et al. (2001) tested a range of grouping cues and demonstrated that the disrupting influence of grouping did not occur for just any connection, but specifically for those conforming to the Gestalt grouping principles of connectedness (Palmer and Rock 1994) or those that formed a plausible object shape. Thus, the reduction in MOT performance caused by grouping targets and distracters is an (indirect) indicator of the strength of a grouping cue, as proven by the influence of the grouping cue on further stages of processing (tracking performance).

We have already mentioned the crucial role of dynamic, participant-guided attention during MOT performance: an MOT task requires keeping track of moving targets. Motion perception problems are reported in ASD, using a quite heterogeneous set of paradigms, and yielding rather mixed results. Increased motion coherence thresholds (Milne et al. 2002; Pellicano et al. 2005; Spencer et al. 2000; but also see De Jonge et al. 2007; Del Viva et al. 2006) and impaired second-order (texture-based) motion detection problems are demonstrated in ASD, despite preserved first-order (luminance-based) motion detection (Bertone et al. 2003). Parameters such as movement speed appear to have a differential impact on motion perception in children with and without ASD (Manning et al. 2013). Extracting coherent motion from noisy input proved difficult for children with ASD when dots were presented at slower speed $(1.5 \% \mathrm{~s})$, but not for fast-moving dots $(6 \%)$. However, this effect did not apply to all motion perception paradigms, as no differential impact of movement speed was found in a speed-discrimination task (Manning et al. 2013). Inspired by these findings, Koldewyn et al. (2012) evaluated the impact of different movement speeds (between $6^{\circ}$ and $29^{\circ} \mathrm{s}$ ) on MOT performance in children with and without ASD. Despite the overall diminished tracking capacity in ASD, Koldewyn et al. (2012) did not obtain evidence for a disproportionally larger deficit at lower (or higher) speeds in ASD. In general, the developmental trajectory of MOT performance seemed to be similar in children with and without ASD, with attentional capacity improvements accounting for better MOT skills (also see Trick et al. 2005; O'Hearn et al. 2010, 2013), and the development of tracking performance at faster speeds improving from the age of seven onwards (Koldewyn et al. 2012).

So far, the modified MOT paradigm (Scholl et al. 2001) has been used mainly to demonstrate the automatic and detrimental influence of grouping cues on tracking performance in adults. O'Hearn et al. (2013) recently showed that grouping based on common motion influences the MOT performance of children too. They asked children to track targets which were coupled with another target (grouping-helps condition) or with a distracter (groupinghurts condition), with items within pairs following the same movement path. Whereas the grouping-helps condition caused a significant increase in tracking performance in comparison to independently moving dots, participants' performance decreased in the grouping-hurts condition (O'Hearn et al. 2013). A first aim of our study was to evaluate whether grouping targets and distracters via a connecting line would also have an interfering (hurting) effect in children, as in adults. Developmental research has suggested that the sensitivity for most Gestalt-based grouping principles increases with development, even for those principles which are already present in infants (Hadad and Kimchi 2006; Hadad et al. 2010a, b; Kovacs 2000; Quinn and Bhatt 2013). In addition, grouping tasks requiring more attentional resources and effort, such as grouping a small number of elements into one structure, show particular age-related improvements (Kimchi et al. 2005).

Secondly, applying a modified MOT task with grouped targets and distracters to children with ASD, allowed us to evaluate the automatic and detrimental effect of grouping cues on MOT performance in this clinical sample. Given the large inconsistencies in the literature on local-global visual processing in ASD, it is pertinent to highlight an important feature of these MOT tasks. In contrast to other tasks, which explicitly instruct participants to attend the 
global level (such as the block design task, object identification tasks, or the classical Gestalt perception tasks), the modified MOT task implicitly measures the influence of grouping via tracking performance. A reduction in MOT performance, caused by a connection between local elements (targets and distracters), indicates grouping strength. The implicit character of this grouping sensitivity measure could minimize the use of potential differences in higherlevel (compensation) strategies, a possible explanation for contradictions in this field (e.g., Plaisted et al. 1999).

In line with the conceptual shift in the literature from a global processing deficit towards a reduced global processing bias, we predicted that the ASD group would show a smaller interfering effect of grouping in comparison to the typically developing (TD) group. This finding would be interesting, since relative improvements are more informative than deficits. Whereas a decreased performance could derive from many potential confounding causes (relative) improvements are more likely to offer an insight into the characteristics of a given disorder (e.g., Shah and Frith 1983). On the other hand, not finding a reduced grouping interference effect on tracking performance might be in line with the recent findings from O'Hearn et al. (2013), demonstrating that children with ASD are equally sensitive to common-fate-based grouping as TD children. Thus, by evaluating the effect of connectedness-based grouping on MOT performance, we were able to investigate the strength of another Gestalt principle in ASD, which parallels the characterization of the differential sensitivity for perceptual grouping phenomena in ASD (Bölte et al. 2007; Brosnan et al. 2004; Falter et al. 2010; Farran and Brosnan 2011).

We compared tracking performance of 6-10 year old children with and without ASD under two MOT conditions: a standard ungrouped condition and a grouped condition, with target-distracter pairs connected via a single line. Applying this modified MOT paradigm in children with and without ASD offers an insight into the influence of objects on attention in both typical and atypical development. In addition, it informs us about the impact of grouping processes in ASD, and moreover, it provides a window into the principles by which sensory input is organized into objects in both participant groups. Therefore, the current study had a twofold aim. First, we investigated whether connection-based grouping has a detrimental influence on MOT performance in children, as reported in adults. Second, we evaluated whether children with ASD are less disrupted by the implicit grouping of targets with distracters than TD children. If so, the performance difference between grouped and ungrouped trials should be smaller in the ASD sample, compared to the TD group. No interaction between grouping condition and participant, on the other hand, would indicate that the detrimental influence of grouping on performance would be essentially the same in both participant groups.

\section{Methods}

\section{Participants}

With written and informed consent from their parent(s), two groups of 6-10-year old children without an intellectual disability (intelligence score within the typical range: FSIQ, PIQ and VIQ $\geq 70$ ) were tested. The ASD group consisted of 18 children, diagnosed by a child psychiatrist or a multidisciplinary team according to DSM-IV-TR criteria (American Psychiatric Association 2000). These children were recruited via the Autism Expertise Centre of the University Hospital $(n=13)$, and via a special needs school for children with ASD $(n=5)$. In accordance with the skewed gender ratio in ASD (e.g., Elsabbagh et al. 2012), significantly more boys than girls with ASD participated in this study (see Table 1). ASD diagnoses were re-evaluated within the research protocol, using the Autism Observation Scale (ADOS; Gotham et al. 2007), which was conducted by a trained clinical psychologist. Seventeen of the 18 children with a clinical ASD diagnosis scored above the ADOS cut-off criterion, with the average ADOS severity score being 5.61. The typically developing (TD) group comprised 39 children recruited via mainstream schools. None of the TD children suffered from a know child psychiatric disorder, nor did any of them had a firstdegree family member with ASD. In addition, ASD traits were measured with the Dutch version of the Social Responsiveness Scale (SRS; Roeyers et al. 2012), with an average raw score far below T70 in the TD group (T70 refers to a norm score of $2 \mathrm{SD}$ above the mean, corresponding to a raw total score of 60 for boys and of 55 for girls). All participants reported normal or corrected-tonormal vision and none of them were taking neuroleptics. In both participant groups, intelligence was assessed with an abbreviated version (Sattler 2001) of the Wechsler Intelligence Scale for Children, Third Edition (WISC-IIINL; Wechsler 1992), which comprised the following subtests: vocabulary, block design, picture completion, and similarities. A subsample of $33 \mathrm{TD}$ children was selected to group-wise match the ASD group on age, intelligence level and gender ratio. Demographic details of both the ASD group and the TD group are shown in Table 1 .

\section{Stimuli and Procedure}

Participants were tested individually, with a viewing distance of approximately $57 \mathrm{~cm}$ from a 17 inch CRT screen (set to pixel resolution $1,024 \times 768$ ). The stimuli and 
Table 1 Descriptive statistics and $p$ values of a two-sample $t$ test (comparison ASD group and matched TD group) with a two-tailed distribution (homoskedasticity assumption checked with $F$ test) for age (depicted in months), performance, verbal and full-scale IQ score (PIQ, VIQ, and FSIQ), SRS total scores, and ADOS severity scores for both participant groups

\begin{tabular}{|c|c|c|c|c|c|c|c|}
\hline & \multicolumn{2}{|c|}{$\begin{array}{l}\text { ASD group ( } n=18 ; 2 \text { girls, } 16 \\
\text { boys) }\end{array}$} & \multicolumn{2}{|c|}{$\begin{array}{l}\text { Whole TD group ( } n=39 ; 10 \text { girls, } 29 \\
\text { boys) }\end{array}$} & \multicolumn{2}{|c|}{$\begin{array}{l}\text { Matched TD group ( } n=33 ; 4 \text { girls, } 29 \\
\text { boys) }\end{array}$} & \multirow{2}{*}{$\begin{array}{l}\text { Two-sided } t \text { test } \\
p \text { value }\end{array}$} \\
\hline & Mean (range) & $\mathrm{SD}$ & Mean & SD & Mean (range) & SD & \\
\hline Age & $103.94(83-123)$ & 13.42 & $101.10(78-124)$ & 15.58 & $101.06(78-123)$ & 14.17 & .4827 \\
\hline VIQ & $104.50(81-135)$ & 12.95 & $111.41(78-135)$ & 12.46 & $109.79(78-135)$ & 12.83 & .1671 \\
\hline PIQ & $105.56(80-134)$ & 14.71 & $105.82(83-134)$ & 11.94 & $105.58(83-134)$ & 12.87 & .9960 \\
\hline FSIQ & $105.03(89-130.5)$ & 11.65 & $108.62(83.5-128)$ & 9.97 & $107.68(83.5-128)$ & 10.56 & .4037 \\
\hline $\mathrm{SRS}^{\mathrm{a}}$ & $93.94(44-135)$ & 20.74 & $30.41(8-100)$ & 19.04 & $32.57(13-100)$ & 20.10 & $<.0001$ \\
\hline ADOS & $5.61(3-9)$ & 1.79 & N/A & N/A & N/A & N/A & N/A \\
\hline
\end{tabular}

${ }^{a}$ SRS data of five TD children and one child with ASD were missing or incomplete

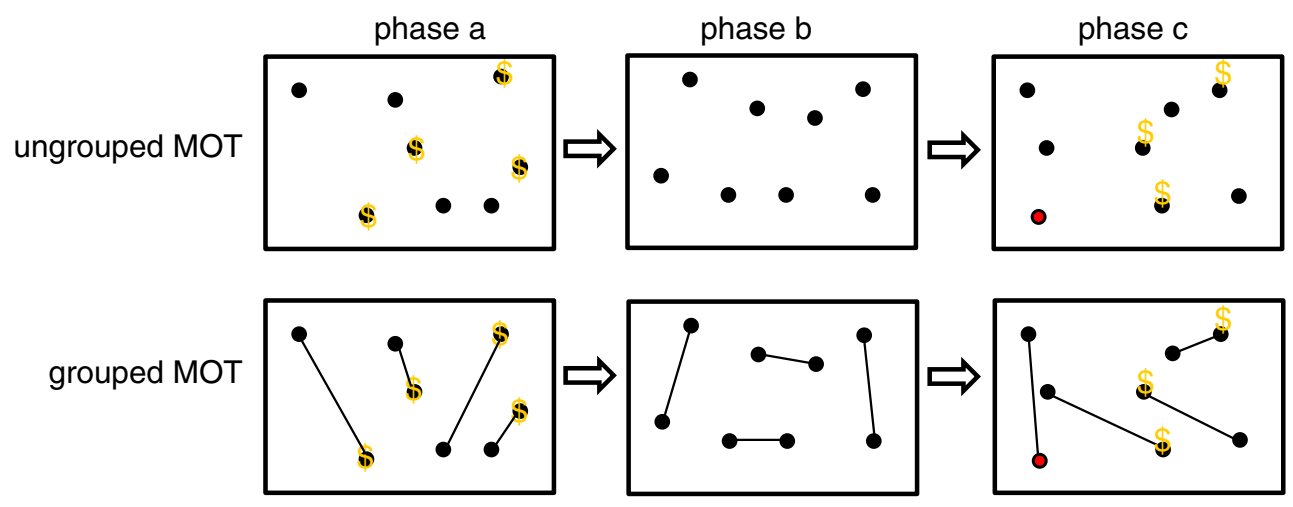

Fig. 1 Illustration of the procedure for multiple object tracking (MOT), which could be divided in three phases. a The four targets were first highlighted with flashing dollar signs. b Participants then had to track these four targets as they moved randomly among distracters. c When the objects came to a stop, participants had to

procedure (Fig. 1) were based upon the connected 'dumbbells' condition from Scholl et al. (2001), with additional changes to make the task easier and more 'game' like for children. Displays were created and controlled with custom-made software. Each trial consisted of eight randomly positioned black dots (diameter: $1.25^{\circ}$ ) on a purple background $(28 \times 20 \mathrm{~cm})$. At the start of each trial, four targets were highlighted (by flashing a golden dollar sign for $4 \mathrm{~s}$ ). Next, all eight dots moved across the screen with an average speed of $2.8^{\circ}$ per second $(\mathrm{SD}=.90 \% \mathrm{~s})$, following independently determined random paths (using Bézier curves) to produce a smooth movement. The distance between two dots varied, with occlusion of dots being allowed during movement (thus no 'bumping' or 'collisions'), but the start and end positions were constrained to avoid any overlap. After $8 \mathrm{~s}$ of movement, the dots stopped and participants had to indicate which four of the eight dots were targets, by clicking on those with the mouse (no time limit was used). If the participant successfully identified a click on the four target objects, and were rewarded with 10 points and the reappearance of the dollar sign when correct. If incorrect, the dot turned red. In this example case, the participant correctly identified three out of four dots (Color figure online)

target, a sound ('k-ching') was played, a golden dollar sign was revealed on that dot, and the number ' 10 ' floated out the correctly selected dot, indicating they had 'won' ten points. If a distracter was selected, the dot would turn red (indicating a mistake was made), no points were awarded and no sounds or other visual rewards were presented. When four out of eight dots were selected, the participant could press the space bar to start the next trial. Participants always had to select four dots, even if they had to guess for some of them.

Before commencing the test trials, participants completed four practice trials with six dots (three targets and three distracters) and four practice trials with eight dots (four targets and four distracters), in order to let the children ease into the task, and to ensure that they understood the instructions. Children were instructed to carefully track the indicated target dots, moving amongst the distracter dots, irrespective of the grouping condition. All participants completed 20 test trials with eight dots (four targets 
and four distracters). On half of the trials, the four target dots were connected to the four distracter dots via black lines ('grouped condition'), for the other half of the trials, no such connection was present ('ungrouped condition'). Both trial types (grouped and ungrouped) were presented in random order.

\section{Data and Data Analysis}

Average performance scores and interference scores were analysed with SAS software, version 9.3 (SAS Institute 2011). Average performance scores referred to the average number of correctly identified targets, with a maximum of four and a chance level of two. Interference scores were calculated by subtracting the average score on the grouped trials from the average score on the ungrouped trials, such that a high interference score corresponded with a large effect of grouping. No outliers were detected for performance scores, and two outliers were detected for interference scores, namely one TD participant scoring more than two standard deviations (SDs) above the group's mean, and one TD participant scoring more than two SDs below the mean. As the analyses with or without outliers yielded the same results, the outliers were included in the reported analyses.

Previous MOT studies in ASD (Koldewyn et al. 2012; O'Hearn et al. 2013) have used capacity scores instead of performance scores, estimating the number of successfully tracked targets (Hulleman 2005). More specifically, they have used capacity formulas which account for the issue of sampling without replacement (the chance of picking a target does not remain constant within a trial) and assuming only targets, and no distracters were tracked. In the context of our design, this last assumption seemed illogical given that a distracter's location (implicitly) contains information about the target in half of the trials. Moreover, different perceptual, cognitive and strategic factors were at play here, such as the influence of guessing strategies and implicit rule extraction. It seems quite likely that these factors would have a differential impact on the performance of children with and without ASD, maybe even depending upon the grouping condition. Because we could not make any generally valid assumptions, necessary to be able to come up with a capacity formula, we chose to analyse and reported the raw performance data.

\section{Results}

Mixed ANOVA Analysis

A mixed ANOVA including grouping condition (grouped vs. ungrouped), participant group (ASD vs. TD) and the

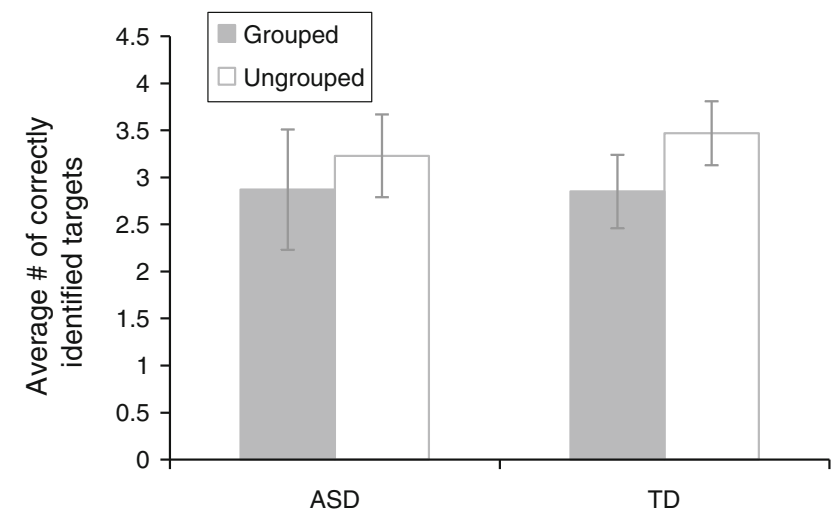

Fig. 2 Significant interaction effect between grouping condition (ungrouped vs. grouped) and participant group (ASD vs. TD) on performance. Error bars represent standard deviations

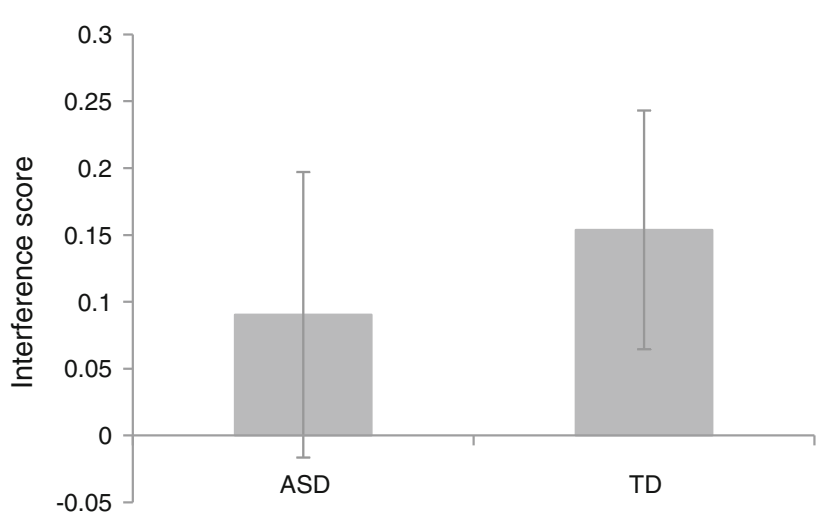

Fig. 3 Significantly reduced grouping interference effect in the ASD group. The interference effect was calculated by subtracting the average score on the grouped trials from the average score on the ungrouped trials, such that a high score corresponds to a large interfering effect of grouping. Error bars represent standard deviations

grouping condition $\times$ participant group interaction as fixed factors, and a random intercept for each subject revealed a main effect of grouping condition $[F(1,49)=75.70$, $p<.0001]$. Specifically, we obtained a clear replication of the interfering impact of linking targets and distracters by line segments (ungrouped condition: $\mathrm{M}=3.38, \mathrm{SD}=.39$; grouped condition: $\mathrm{M}=2.86, \mathrm{SD}=.49$ ). There was no evidence for an overall performance difference between the TD sample and the ASD sample [TD group: $\mathrm{M}=3.16$, $\mathrm{SD}=.48$; ASD group: $\mathrm{M}=3.05, \mathrm{SD}=.57 ; F(1,49)=$ $.99, p=.3245]$. Most interestingly, there was an interaction between participant group and grouping condition $[F(1,49)=5.13, p=.0280$, see Fig. 2], consistent with the prediction that participants with ASD are less affected by the grouping of targets and distracters. However, the effect of grouping condition remained clearly noticeable within both the TD and the ASD sample (simple main 


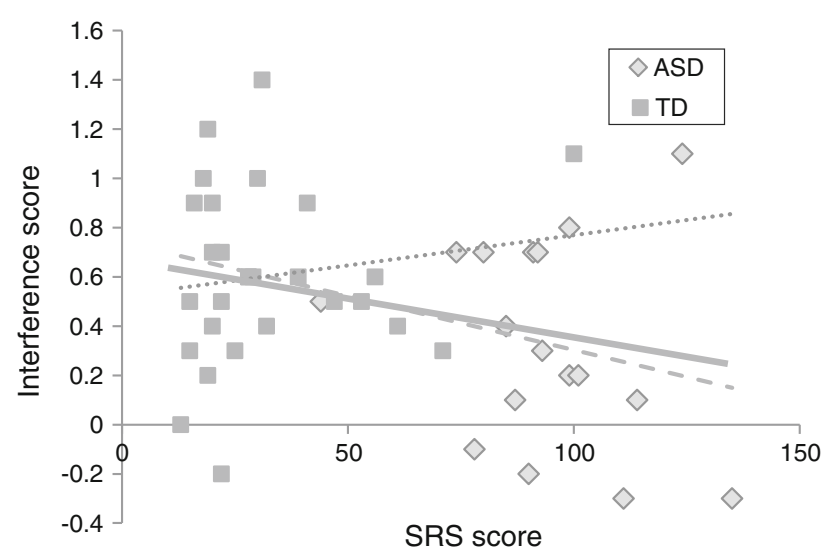

Fig. 4 Interference scores for both matched groups plotted against SRS total scores, to show the relationship between ASD traits and interference scores. The full line shows the linear trend across both participant groups, the dotted line represents the linear trend within the TD group, and the dashed line within the ASD group

effect analysis, both with $p$ value <.0001). Controlling for age, TIQ or both did not change our results. To further evaluate and interpret this interaction effect, interference scores were analyzed.

\section{Interference Scores}

A two sample $t$ test $[t(49)=-2.26, p=.0280]$, equal variances assumed; Levene's test: $F(1,49)=.99$, $p=.3235)$ showed that the TD group suffered from a larger interfering impact of grouping targets and distracters than the ASD group (see Fig. 3: TD group: $\mathrm{M}=.15$, $\mathrm{SD}=.09 ;$ ASD group: $\mathrm{M}=.09 ; \mathrm{SD}=.11$ ).

\section{Correlations Between Interference Score} and Standardized Measures

Pearson correlations are reported for the whole sample, although a similar pattern of results was found when analysing the ASD and TD groups separately, unless explicitly mentioned. Despite the positive significant correlation between age and MOT performance $(r=.44$, $p=.0013)$, no evidence for a correlation between age and interference score was found $(r=-.11, p=.4293)$. Both interference scores and average performance were not correlated with TIQ measures $(p>.55)$. No evidence for a correlation between overall MOT performance and SRS score was found $(r=-.17, p=.2610)$, but interference scores tended to be negatively correlated with SRS scores (across groups correlation: Pearson $r=-.28$, $p=.0581$; see Fig. 4). Children with a higher SRS score (i.e., more ASD traits) had a lower interference score (i.e., smaller influence of grouping). Note, however, that ASD traits (SRS scores) only account for a small proportion of the variability in the interference scores, as indicated by the coefficients of determination $\left(R^{2}\right.$ values). Within-group correlations between interference score and SRS were not significant (ASD: $r=-.22$, $p=.4016$; TD: $r=.14, p=.4825$ ), probably due to the smaller number of participants, and the limited SRS range in the TD group.

\section{Discussion}

First of all, the current study demonstrates that the mandatory influence of grouping on MOT is evident in children aged $6-10$, as previously reported in adults (Scholl et al. 2001). This strengthens the idea that (object-based) grouping plays a key role in shaping the sensory information process throughout the whole lifespan (Carey and $\mathrm{Xu}$ 2001). Moreover, it complements findings by O'Hearn et al. (2013), showing that the tracking performance of children aged 9-12 was influenced by common motionbased grouping, to the same extent as adolescents and adults. All together, these results demonstrate that also in children, grouping local elements into larger units (grouped targets and distracters) influences the access to the separate local elements.

Koldewyn et al. (2012) evaluated the impact of movement speed on tracking performance in 5-12-year old children with and without ASD. Despite the absence of an atypical effect of speed, the ASD sample generally showed a reduced MOT performance in comparison to the TD sample. O'Hearn et al. (2013) demonstrated an overall poorer tracking performance in children with ASD in three MOT conditions: the ungrouped condition, the target-target grouping condition, and the target-distracter grouping condition, using common motion as grouping cue. In line with these recent studies, our ASD sample performed worse than the TD group in the ungrouped MOT condition. We, however, did not find an overall poorer tracking performance.

In the ASD sample too, the influence of grouping upon object tracking is clearly present. However, the interfering effect of connecting targets and distracters was significantly reduced in the ASD group. The present, but reduced impact of grouping on tracking performance in ASD supports the recent shift in neurocognitive theories, from a global deficit approach towards a disinclination for global or an increased preference for local processing. As already noted in the "Introduction" section, the modified MOT paradigm implicitly measures grouping strength, via its influence on tracking performance. An implicit measurement has the advantage of minimizing the use of potential higher-level (compensation) strategies, a possible explanation for contradictions in this field (Plaisted et al. 1999). 
Although our results cannot differentiate between a reduced process of integration or a preferential allocation of attention to earlier (less integrated) stages of sensory processing, they do demonstrate that the manner in which attention selects information is altered in children with ASD. The default allocation of processing resources to whole objects versus local parts had an important influence on further stages of perception and cognition. It can therefore be interesting to reflect on the potential changes this might have, in particular in the context of joint attention. Joint attention is a behavioural pattern where attention for an object (or person) of interest is shared between two individuals, and forms a crucial ability in social interactions (Scaife and Bruner 1975). If attention is less automatically influenced by grouping cues in contrived laboratory conditions, as in MOT tracking, it is not unlikely that it will also affect social ways of guiding attention in more complex, ecologically valid situations. This connection with everyday life remains strongly speculative, but receives support from the fact that interference scores tended to be correlated with SRS scores, indicating that participants with more ASD traits showed a smaller interfering effect of grouping. Recently, the traditional, categorical view on ASD is challenged by a more quantitative approach (e.g., Constantino and Todd 2003). It indeed appears that we found a relationship between ASD traits and a more locally oriented perceptual style (see also e.g., Reed et al. 2011). However, these results should be interpreted with caution, given the small effect size of these correlations, and the lack of clear support from the withingroup correlations (as this effect was driven by an association within the ASD sample that was not present in the TD sample).

All together, the current results demonstrate that grouping also hampers tracking performance in children with ASD. However, the global context (connecting targets and distracters) seems to affect the tracking of the local elements (targets) to a lesser extent in ASD. These results contrast with the recent findings by O'Hearn et al. (2013), showing a typical influence of common fate-based grouping on MOT performance. Although both studies generally used similar parameters (e.g., number of targets and distracters, target size, occlusion of objects), some differences could be noted. For example, O'Hearn et al. (2013) used a faster movement speed, and they showed proportionally more grouped than ungrouped trials, which could have an effect on cognitive and strategic processes. However, the most likely reason for these contradicting findings is a differential sensitivity for both grouping principles in ASD. Up to now, research about the sensitivity for different Gestalt principles in ASD presents a heterogeneous picture, caused by differences in participants' age, intelligence levels, and the diversity of evaluated principles.
Whereas grouping based on similarity (Bölte et al. 2007; Falter et al. 2010; Farran and Brosnan 2011) or closure (Bölte et al. 2007; Brosnan et al. 2004) is mostly disturbed in ASD, the evidence on proximity-based grouping is mixed, with some researchers finding a diminished sensitivity (Bölte et al. 2007; Brosnan et al. 2004), and other studies suggesting typical levels of proximity-based grouping (Falter et al. 2010; Farran and Brosnan 2011). Comparing our results with O'Hearn et al.'s findings (2013), the influence of common-motion-based grouping appears unaffected in ASD, despite a reduced sensitivity for connection-based grouping. In addition, grouping can also have a positive impact on tracking performance under certain circumstances, e.g., by connecting targets with targets, and distracters with distracters (O'Hearn et al. 2013). It would be interesting to evaluate whether this also holds for other grouping principles. Future research with the modified MOT paradigm allows for a direct comparison of the impact of different Gestalt principles on tracking performance between individuals with and without ASD. We did not find indications for an increasing impact of this grouping phenomenon with age (also see O'Hearn et al. 2013). Here too, a direct comparison of the developmental trajectories between children with and without developmental disorder would be valuable.

Acknowledgments We are grateful to all participating children and their parents. Special thanks to the participating schools, Ten Bunderen (Moorslede), De Puzzel (Kleine-Brogel) and De Schommel (Lommel). We thank the following master students for their assistance with data collection: Nele Berghmans, Sanne Drees, Tine Herreman, Ellen Janssen, and Lotte Van Esch. We want to acknowledge professor Johan Hulleman for his advice concerning data analysis. This research was funded by the Methusalem program by the Flemish Government (METH 08/02) awarded to Johan Wagemans and a grant from the Research Council of the KU Leuven (IDO/080/013) awarded to Jean Steyaert, Johan Wagemans, and Ilse Noens.

\section{References}

American Psychiatric Association. (2000). Diagnostic and statistical manual of mental disorders (4th edition text revision). Washington, DC: Author.

Behrmann, M., Thomas, C., \& Humphreys, K. (2006). Seeing it differently: Visual processing in autism. Trends in Cognitive Sciences, 10(6), 258-264. doi:10.1016/j.tics.2006.05.001.

Bertone, A., Mottron, L., Jelenic, P., \& Faubert, J. (2003). Motion perception in autism: A "complex" issue. Journal of Cognitive Neuroscience, 15(2), 218-225. doi:10.1162/089892903321208150.

Bölte, S., Holtmann, M., Poustka, F., Scheurich, A., \& Schmidt, L. (2007). Gestalt perception and local-global processing in highfunctioning autism. Journal of Autism and Developmental Disorders, 37, 1493-1504.

Brosnan, M. J., Scott, F. J., Fox, S., \& Pye, J. (2004). Gestalt processing in autism: Failure to process perceptual relationships and the implications for contextual understanding. Journal of Child Psychology and Psychiatry, 45(3), 459-469. 
Carey, S., \& Xu, F. (2001). Infants' knowledge of objects: Beyond object files and object tracking. Cognition, 80(1-2), 179-213. doi:10.1016/S0010-0277(00)00154-2.

Constantino, J. N., \& Todd, R. D. (2003). Autistic traits in the general population-A twin study. Archives of General Psychiatry, 60(5), 524-530. doi:10.1001/archpsyc.60.5.524.

Dakin, S., \& Frith, U. (2005). Vagaries of visual perception in autism. Neuron, 48, 497-507. doi:10.1016/j.neuron.2005.10.018.

De Jonge, M. V., Kemner, C., De Haan, E. H., Coppens, J. E., Van Den Berg, T. J. T. P., \& Van Engeland, H. (2007). Visual information processing in high-functioning individuals with autism spectrum disorders and their parents. Neuropsychology, 21(1), 65-73.

Del Viva, M. M., Igliozzi, R., Tancredi, R., \& Brizzolara, D. (2006). Spatial and motion integration in children with autism. Vision Research, 46(8-9), 1242-1252. doi:10.1016/j.visres.2005.10. 018.

Driver, J., Davis, G., Russell, C., Turatto, M., \& Freeman, E. (2001). Segmentation, attention and phenomenal visual objects. Cognition, 80(1-2), 61-95.

Elsabbagh, M., Divan, G., Koh, Y.-J., Kim, Y. S., Kauchali, S., Marcín, C., et al. (2012). Global prevalence of autism and other pervasive developmental disorders. Autism Research, 5(3), 160-179. doi:10.1002/aur.239.

Falter, C. M., Grant, K. C. P., \& Davis, G. (2010). Object-based attention benefits reveal selective abnormalities of visual integration in autism. Autism Research, 3(3), 128-136. doi:10.1002/ aur.134.

Farran, E. K., \& Brosnan, M. J. (2011). Perceptual grouping abilities in individuals with autism spectrum disorder: Exploring patterns of ability in relation to grouping type and levels of development. Autism Research, 4(4), 283-292. doi:10.1002/aur.202.

Frith, U., \& Happé, F. (1994). Autism-Beyond theory of mind. Cognition, 50(1-3), 115-132. doi:10.1016/0010-0277(94) 90024-8.

Gotham, K., Risi, S., Pickles, A., \& Lord, C. (2007). The autism diagnostic observation schedule: Revised algorithms for improved diagnostic validity. Journal of Autism and Developmental Disorders, 37(4), 613-627. doi:10.1007/s10803-0060280-1.

Hadad, B., \& Kimchi, R. (2006). Developmental trends in utilizing perceptual closure for grouping of shape: Effects of spatial proximity and collinearity. Perception \& Psychophysics, 68(8), 1264-1273. doi:10.3758/BF03193726.

Hadad, B., Maurer, D., \& Lewis, T. L. (2010a). The development of contour interpolation: Evidence from subjective contours. Journal of Experimental Child Psychology, 106(2-3), 163-176. doi:10.1016/j.jecp.2010.02.003.

Hadad, B., Maurer, D., \& Lewis, T. L. (2010b). The effects of spatial proximity and collinearity on contour integration in adults and children. Vision Research, 50(8), 772-778. doi:10.1016/j.visres. 2010.01.021.

Happé, F. (1996). Studying weak central coherence at low levels: Children with autism do not succumb to visual illusions. A research note. Journal of Child Psychology and Psychiatry and Allied Disciplines, 37(7), 873-877. doi:10.1111/j.1469-7610. 1996.tb01483.x.

Happé, F., \& Booth, R. D. L. (2008). The power of the positive: Revisiting weak coherence in autism spectrum disorders. The Quarterly Journal of Experimental Psychology, 61(1), 50-63. doi:10.1080/17470210701508731.

Happé, F., \& Frith, U. (2006). The weak coherence account: Detailfocused cognitive style in autism spectrum disorders. Journal of Autism and Developmental Disorders, 36(1), 5-25. doi:10.1007/ s10803-005-0039-0.
Hulleman, J. (2005). The mathematics of multiple object tracking: From proportions correct to number of objects tracked. Vision Research, 45(17), 2298-2309. doi:10.1016/j.visres.2005.02.016.

Kimchi, R., Hadad, B., Behrmann, M., \& Palmer, S. E. (2005). Microgenesis and ontogenesis of perceptual organizationEvidence from global and local processing of hierarchical patterns. Psychological Science, 16(4), 282-290. doi:10.1111/j. 0956-7976.2005.01529.x.

Koldewyn, K., Jiang, Y. V., Weigelt, S., \& Kanwisher, N. (2013). Global/local processing in autism: Not a disability, but a disinclination. Journal of Autism and Developmental Disorders, . doi:10.1007/s10803-013-1777-z.

Koldewyn, K., Weigelt, S., Kanwisher, N., \& Jiang, Y. (2012). Multiple object tracking in autism spectrum disorders. Journal of Autism and Developmental Disorders, . doi:10.1007/s10803-0121694-6.

Kovacs, I. (2000). Human development of perceptual organization. Vision Research, 40(10-12), 1301-1310. doi:10.1016/S00426989(00)00055-9.

Loth, E., Gomez, J. C., \& Happé, F. (2010). When seeing depends on knowing: Adults with autism spectrum conditions show diminished top-down processes in the visual perception of degraded faces but not degraded objects. Neuropsychologia, 48(5), 1227-1236. doi:10.1016/j.neuropsychologia.2009.12.023.

Manning, C., Charman, T., \& Pellicano, E. (2013). Processing slow and fast motion in children with autism spectrum conditions. Autism Research,. doi:10.1002/aur.1309.

Milne, E., Swettenham, J., Hansen, P., Campbell, R., Jeffries, H., \& Plaisted, K. (2002). High motion coherence thresholds in children with autism. Journal of Child Psychology and Psychiatry, 43(2), 255-263.

Mitchell, P., Mottron, L., Soulieres, I., \& Ropar, D. (2010). Susceptibility to the Shepard illusion in participants with autism: Reduced top-down influences within perception? Autism Research, 3(3), 113-119. doi:10.1002/aur.130.

Mottron, L., Bouvet, L., Bonnel, A., Samson, F., Burack, J. A., Dawson, M., et al. (2013). Veridical mapping in the development of exceptional autistic abilities. Neuroscience and Biobehavioral Reviews, 37(2), 209-228. doi:10.1016/j.neubiorev.2012.11.016.

Mottron, L., \& Burack, J. A. (2001). Enhanced perceptual functioning in the development of autism. In J. A. Burack, T. Charman, N. Yirmiya, \& P. R. Zelazo (Eds.), The development of autism: Perspectives from theory and research (pp. 131-148). Mahwah, NJ: Erlbaum.

Mottron, L., Dawson, M., Soulières, I., Hubert, B., \& Burack, J. (2006). Enhanced perceptual functioning in autism: An update, and eight principles of autistic perception. Journal of Autism and Developmental Disorders, 36(1), 27-43. doi:10.1007/s10803005-0040-7.

O’Hearn, K., Franconeri, S., Wright, C., Minshew, N., \& Luna, B. (2013). The development of individuation in autism. Journal of Experimental Psychology: Human Perception and Performance, 39(2), 494-509. doi:10.1037/a0029400.

O'Hearn, K., Hoffman, J. E., \& Landau, B. (2010). Developmental profiles for multiple object tracking and spatial memory: Typically developing preschoolers and people with Williams syndrome. Developmental Science, 13(3), 430-440. doi:10.1111/ j.1467-7687.2009.00893.x.

Palmer, S., \& Rock, I. (1994). Rethinking perceptual organizationThe role of uniform connectedness. Psychonomic Bulletin \& Review, 1(1), 29-55. doi:10.3758/BF03200760.

Pellicano, E., Gibson, L., Maybery, M., Durkin, K., \& Badcock, D. R. (2005). Abnormal global processing along the dorsal visual pathway in autism: A possible mechanism for weak visuospatial coherence? Neuropsychologia, 43, 1044-1053. 
Plaisted, K., Swettenham, J., \& Rees, L. (1999). Children with autism show local precedence in a divided attention task and global precedence in a selective attention task. Journal of Child Psychology and Psychiatry, 40(5), 733-742.

Pylyshyn, Z. W., \& Storm, R. W. (1988). Tracking multiple independent targets: Evidence for a parallel tracking mechanism. Spatial Vision, 3(3), 179-197. doi:10.1163/156856888X00122.

Quinn, P. C., \& Bhatt, R. S. (2013). Development of perceptual organization in infancy. In J. Wagemans (Ed.), Oxford handbook of perceptual organization. Oxford: Oxford University Press (in press).

Reed, P., Lowe, C., \& Everett, R. (2011). Perceptual learning and perceptual search are altered in male university students with higher autism quotient scores. Personality and Individual Differences, 51(6), 732-736. doi:10.1016/j.paid.2011.06.016.

Roeyers, H., Thys, M., Druart, C., De Schryver, M., \& Schittekatte, M. (2012). SRS: Screeningslijst voor autismespectrum stoornissen. Amsterdam: Hogrefe.

Samson, F., Mottron, L., Soulieres, I., \& Zeffiro, T. A. (2012). Enhanced visual functioning in autism: An ALE meta-analysis. Human Brain Mapping, 33(7), 1553-1581. doi:10.1002/hbm. 21307.

SAS Institute. (2011). SAS software, version 9.3 of the SAS system for Microsoft Windows. Cary, NC: SAS Institute.

Sattler, J. M. (2001). Assessment of children: Cognitive applications (4th ed.). San Diego, CA: Jerome M. Sattler.

Scaife, M., \& Bruner, J. S. (1975). The capacity for joint visual attention in the infant. Nature, 253(5489), 265-266. doi:10.1038/ $253265 \mathrm{a} 0$.

Scholl, B. J., Pylyshyn, Z. W., \& Feldman, J. (2001). What is a visual object? Evidence from target merging in multiple object tracking. Cognition, $80(1-2)$, 159-177. doi:10.1016/S00100277(00)00157-8.

Shah, A., \& Frith, U. (1983). An islet of ability in autistic children: A research note. Journal of Child Psychology and Psychiatry and Allied Disciplines, 24, 613-620. doi:10.1111/j.1469-7610.1983. tb00137.x.

Shah, A., \& Frith, U. (1993). Why do autistic individuals show superior performance on the block design task. Journal of Child Psychology and Psychiatry and Allied Disciplines, 34(8), 1351-1364. doi:10.1111/j.1469-7610.1993.tb02095.x.

Simmons, D. R., Robertson, A. E., McKay, L. S., Toal, E., McAleer, P., \& Pollick, F. E. (2009). Vision in autism spectrum disorders. Vision Research, 49(22), 2705-2739. doi:10.1016/j.visres.2009. 08.005 .

Spencer, J., O’Brien, J., Riggs, K., Braddick, O., Atkinson, J., \& Wattam-Bell, J. (2000). Motion processing in autism: Evidence for a dorsal stream deficiency. NeuroReport, 11(12), 2765-2767.

Trick, L. M., Jaspers-Fayer, F., \& Sethi, N. (2005). Multiple-object tracking in children: The "catch the spies" task. Cognitive Development, 20(3), 373-387. doi:10.1016/j.cogdev.2005.05.009.

Tsatsanis, K. D., Noens, I. L. J., Illmann, C. L., Pauls, D. L., Volkmar, F. R., Schultz, R. T., et al. (2011). Managing complexity: Impact of organization and processing style on nonverbal memory in autism spectrum disorders. Journal of Autism and Developmental Disorders, 41(2), 135-147. doi:10.1007/s10803-010-1139-z.

Wechsler, D. (1992). Wechsler intelligence scale for children (3rd ed.). London: The Psychological Corporation.

White, S. J., \& Saldana, D. (2011). Performance of children with autism on the embedded figures test: A closer look at a popular task. Journal of Autism and Developmental Disorders, 41(11), 1565-1572. doi:10.1007/s10803-011-1182-4. 